Case Report

\title{
Sanguineous Pericardial Effusion and Cardiac Tamponade in the Setting of Graves' Disease: Report of a Case and Review of Previously Reported Cases
}

\author{
Peter V. Bui, Sonia N. Zaveri, and J. Rush Pierce Jr. \\ Department of Internal Medicine, The University of New Mexico, Albuquerque, NM 87106, USA \\ Correspondence should be addressed to J. Rush Pierce Jr.; jrpierce@salud.unm.edu
}

Received 20 March 2016; Accepted 5 June 2016

Academic Editor: Grigrios Korosoglou

Copyright (C) 2016 Peter V. Bui et al. This is an open access article distributed under the Creative Commons Attribution License, which permits unrestricted use, distribution, and reproduction in any medium, provided the original work is properly cited.

\begin{abstract}
Introduction. Pericardial effusion in the setting of hyperthyroidism is rare. We present a patient with Graves' disease who developed a sanguineous pericardial effusion and cardiac tamponade. Case Description. A 76-year-old man presenting with fatigue was diagnosed with Graves' disease and treated with methimazole. Two months later, he was hospitalized for uncontrolled atrial fibrillation. Electrocardiography showed diffuse low voltage and atrial fibrillation with rapid ventricular rate. Chest radiograph revealed an enlarged cardiac silhouette and left-sided pleural effusion. Thyroid stimulating hormone was undetectable, and free thyroxine was elevated. Diltiazem and heparin were started, and methimazole was increased. Transthoracic echocardiography revealed a large pericardial effusion with cardiac tamponade physiology. Pericardiocentesis obtained 1,050 mL of sanguineous fluid. The patient progressed to thyroid storm, treated with propylthiouracil, potassium iodine, hydrocortisone, and cholestyramine. Cultures and cytology of the pericardial fluid were negative. Thyroid hormone markers progressively normalized, and he improved clinically and was discharged. Discussion. We found 10 previously reported cases of pericardial effusions in the setting of hyperthyroidism. Heparin use may have contributed to the sanguineous nature of our patient's pericardial effusion, but other reported cases occurred without anticoagulation. Sanguineous and nonsanguineous pericardial effusions and cardiac tamponade may be due to hyperthyroidism.
\end{abstract}

\section{Introduction}

Hyperthyroidism is found in $1.3 \%$ of the United States population (12 years and older), and cardiac manifestations are common [1]. However, of those cardiac findings, pericardial effusion associated with hyperthyroidism has rarely been reported, limited to a small number of case reports primarily in the setting of Graves' disease [2-8]. We report a patient with Graves' disease and hyperthyroidism who developed a sanguineous pericardial effusion with cardiac tamponade. We reviewed the literature for previous case reports of patients with hyperthyroidism and pericardial effusion.

\section{Case Description}

A 76-year-old diabetic man presented to an outpatient clinic with fatigue. He was found to have a total thyroxine (T4) of $22.5 \mu \mathrm{g} / \mathrm{dL}$ (normal $4.5-12.1 \mu \mathrm{g} / \mathrm{dL}$ ) and an undetectable thyroid stimulating hormone (TSH) (normal 0.358$3.740 \mathrm{IUI} / \mathrm{mL}$ ). Diagnostic studies for hyperthyroidism found a thyroglobulin antibody of $<20 \mathrm{IU} / \mathrm{mL}$ (normal $<$ $40 \mathrm{IU} / \mathrm{mL}$ ), thyroid peroxidase antibody of $17.0 \mathrm{IU} / \mathrm{mL}$ (normal $<35 \mathrm{IU} / \mathrm{mL}$ ), thyroid stimulating immunoglobulin of $490 \%$ (normal $\leq 122 \%$ ), thyroid stimulating hormone receptor antibody of $3.91 \mathrm{IU} / \mathrm{L}$ (normal $\leq 1.75 \mathrm{IU} / \mathrm{L}$ ), 24-hour radioactive iodine-123 uptake of $45 \%$ (normal 10-30\%) (Figure 1), and multiple smaller-than-one-centimeter thyroid nodules via an ultrasound of the thyroid. Physical examination did not find ophthalmopathy or dermopathy. He was diagnosed with Graves' disease for which he was started on methimazole $10 \mathrm{mg}$ daily. Over the next two months, he developed dyspnea on exertion, insomnia, fevers, night sweats, productive cough, and weight loss. He 


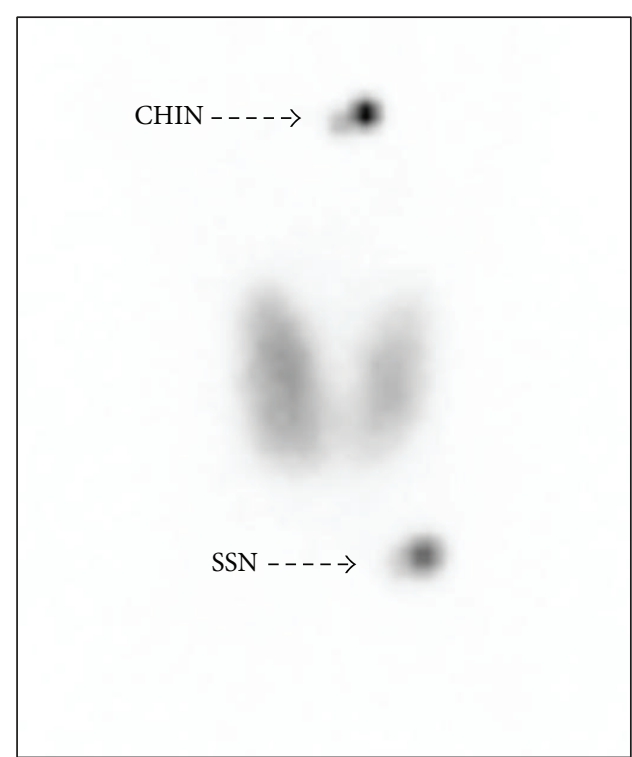

(a)

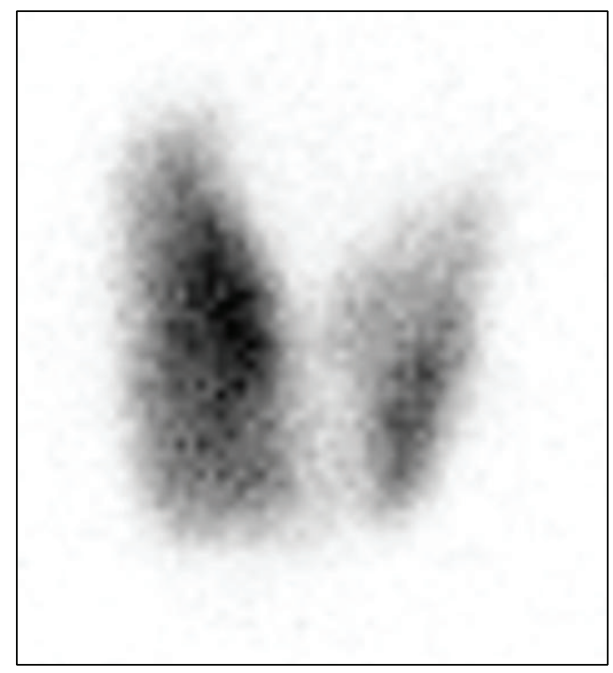

(c)

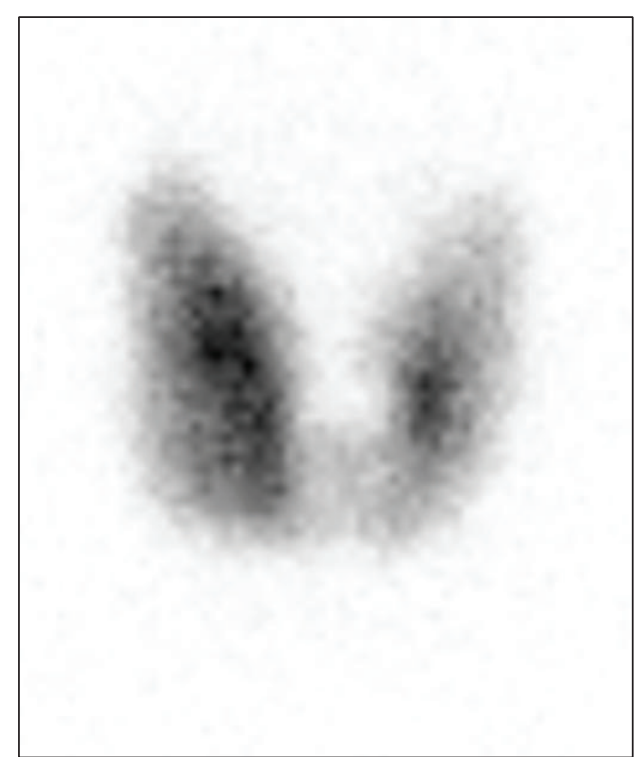

(b)

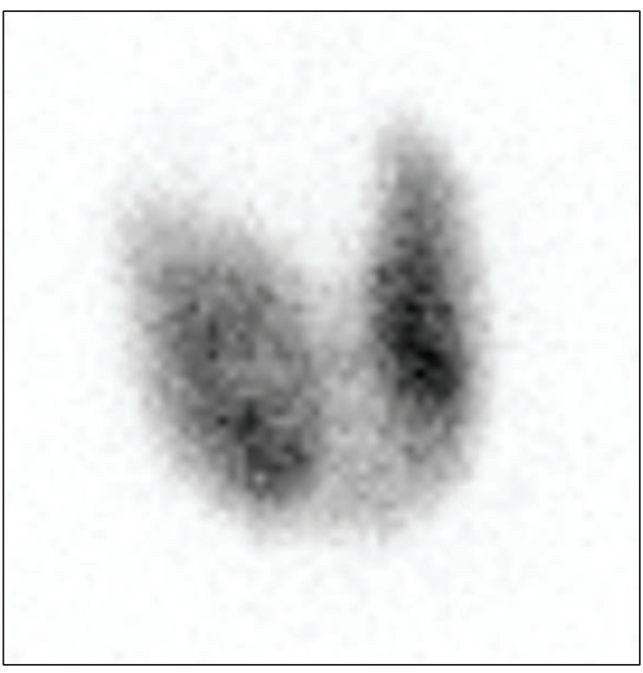

(d)

FIGURE 1: Twenty-four-hour radioactive iodine-123 uptake test. The images show diffuse uptake of $45 \%$ in the anterior view at high resolution with markers (a), anterior view with the pinhole collimator (b), right anterior oblique view with the pinhole collimator (c), and left anterior oblique view with the pinhole collimator (d). SSN: substernal notch.

presented back to the clinic in atrial fibrillation with rapid ventricular rate and was admitted to the hospital.

On the day of admission, conventional chest radiography found an enlarged cardiac silhouette, bibasilar opacities, and left-sided pleural effusion (Figure 2). Cardiac markers included $\mathrm{N}$-terminal prohormone of brain natriuretic peptide of $1,172 \mathrm{pg} / \mathrm{mL}$ (normal 0-450 pg/mL) and two-consecutive troponin $\mathrm{I}<0.017 \mathrm{ng} / \mathrm{mL}$ (normal $0-0.059 \mathrm{ng} / \mathrm{mL}$ ). Thyroid hormone levels were TSH of $0.007 \mathrm{UIU} / \mathrm{mL}$ (normal 0.358-3.740 IUI/mL), free T4 $3.7 \mathrm{ng} / \mathrm{dL}$ (normal 0.7$1.6 \mathrm{ng} / \mathrm{dL}$ ), total T4 $21.3 \mu \mathrm{g} / \mathrm{dL}$ (normal $4.5-12.1 \mu \mathrm{g} / \mathrm{dL}$ ), and free triiodothyronine $235 \mathrm{ng} / \mathrm{dL}$ (normal 55-172 ng/dL). Electrocardiography showed diffuse low voltage and was consistent with atrial fibrillation with rapid ventricular rate.
Methimazole was increased to $30 \mathrm{mg}$ daily. He was started on continuous diltiazem infusion (the patient had an allergy to beta blockers), continuous heparin infusion for atrial fibrillation, and ceftriaxone and azithromycin for possible community-acquired pneumonia.

On hospital day 1 , transthoracic echocardiography (TTE) found a pericardial effusion (Figure 3), mitral wave flow variation of greater than $30 \%$, mild right ventricular diastolic compression, and plethoric inferior vena cava with minimal reactivity. With continuous heparin infusion being discontinued shortly before the procedure, pericardiocentesis with the placement of a pericardial drain drained $850 \mathrm{~mL}$ of sanguineous fluid during the procedure, and $200 \mathrm{~mL}$ of fluid was drained during the subsequent day. Studies of the pericardial 


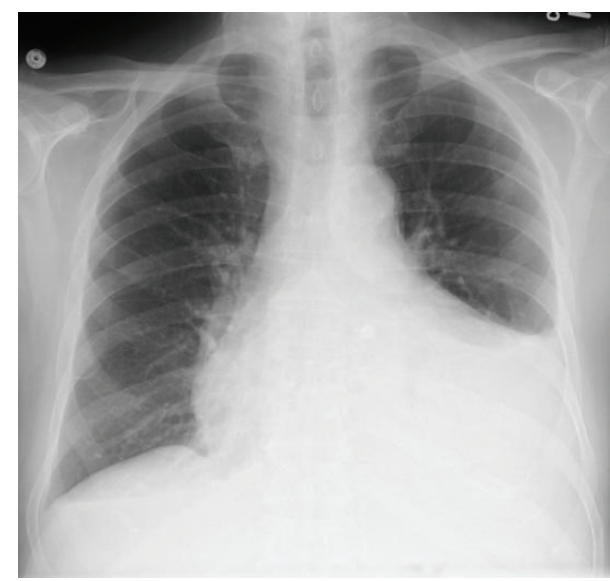

(a)

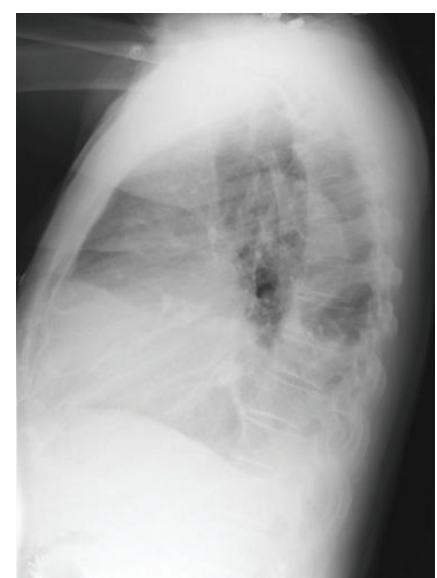

(b)

FIGURE 2: Conventional chest radiograph on the day of admission. The images show an enlarged cardiac silhouette, bibasilar opacities, and left-sided pleural effusion.

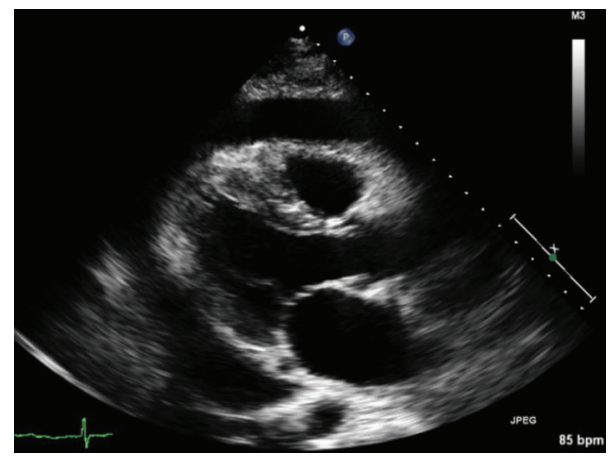

FIGURE 3: Transthoracic echocardiography (TTE) performed on day one of the hospitalization. The image shows a pericardial effusion.

fluid found 607,000 red blood cells (RBCs), 4,548 total nucleated cells, 49\% neutrophils, 15\% lymphocytes, 36\% mononuclear cells, $0 \%$ eosinophils, $0 \%$ other cells, $0 \%$ nucleated cells, glucose of $121 \mathrm{mg} / \mathrm{dL}$ (no reference range), lactate dehydrogenase $(\mathrm{LDH})$ of 1,326 units/L (no reference range), specific gravity of 1.030 (no reference range), and total protein of $4.8 \mathrm{~g} / \mathrm{dL}$ (no reference range). On that subsequent day, continuous diltiazem infusion was transitioned to oral diltiazem and he began aspirin.

On hospital day 3, he experienced worsening dyspnea, fever, and diarrhea consistent with thyroid storm, for which he received propylthiouracil potassium iodide, hydrocortisone, and cholestyramine. A left-sided thoracentesis was performed for increasing dyspnea and oxygen requirement and drained $1,250 \mathrm{~mL}$ of serous fluid. Studies of the pleural fluid found 1,000 RBCs, 364 total nucleated cells, 30\% neutrophils, $44 \%$ lymphocytes, $26 \%$ mononuclear cells, $0 \%$ eosinophils, $0 \%$ other cells, $0 \%$ nucleated red blood cells, glucose of $140 \mathrm{mg} / \mathrm{dL}$ (no reference range), LDH of 78 units/L (no reference range), $\mathrm{pH}$ of 7.432 (no reference range), and total protein of $2.6 \mathrm{~g} / \mathrm{dL}$ (no reference range). Studies were consistent with transudative effusion by Light's criteria. On hospital day 5, the pericardial drain was removed, and TTE two days later showed trivial pericardial effusion. Cultures of the blood, pericardial fluid, pleural fluid, and sputum had no growth. Cytology of the pericardial fluid did not find malignant cells. He converted spontaneously to sinus rhythm and was discharged on methimazole $30 \mathrm{mg}$ orally daily, hydrocortisone $15 \mathrm{mg}$ orally every morning and $10 \mathrm{mg}$ orally every evening, diltiazem $240 \mathrm{mg}$ orally daily, and aspirin $81 \mathrm{mg}$ orally daily. At follow-up clinic visits six weeks and five months later, he was asymptomatic, free T4 was normal, and ECG revealed sinus rhythm and normalization of voltage.

\section{Discussion}

Pericardial effusions occur in approximately 3\% to $6 \%$ of patients with hypothyroidism [9]. Contrastingly, in our review of the literature, we found only 10 previously reported cases of pericardial effusion in the setting of hyperthyroidism (Table 1). Four of the pericardial effusions were sanguineous $[2,5,7,8]$. Similar to our patient, six of the patients had Graves' disease $[2,3,7,8]$. Five of the 10 reported patients had atrial fibrillation [2, 6-8]. Our patient's constellation of sanguineous pericardial effusion and atrial fibrillation treated with continuous heparin infusion appears unique among the available case reports, and the heparin may have contributed to the blood in our patient's pericardial effusion.

The mechanism for the development of a pericardial effusion with Graves' disease has not been elucidated. Previous authors have postulated that the mechanism is similar to that of the ophthalmopathy and myxedema found in hyperthyroidism $[2,5,8]$. A study of hypothyroidism found shifts in extravascular and intravascular proteins and a decrease in lymph drainage [10]. Hyperthyroidism may involve a similar pathophysiology. Given the limited information provided in the available case reports, the contribution of anticoagulation to the sanguineous nature of the pericardial effusion is unknown. For our patient, the presence of RBCs in the pericardial effusion and pleural effusion, albeit a small amount 


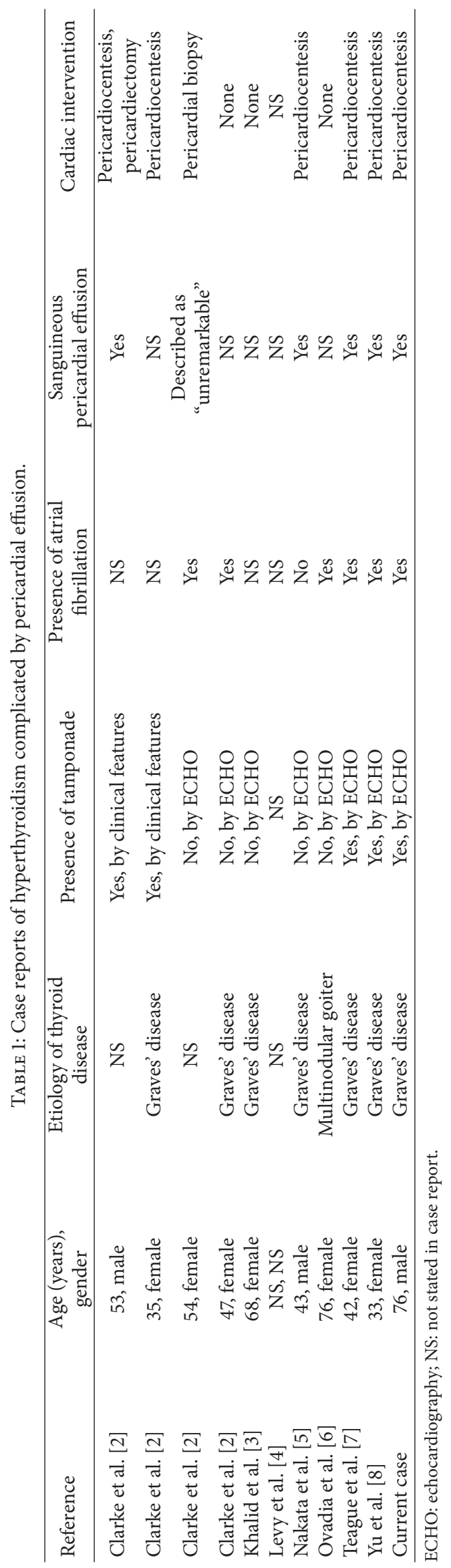


in the pleural effusion, suggested that anticoagulation may be a factor in the development of sanguineous pericardial effusions in the setting of Graves' disease, assuming that the procedures were not notably traumatic.

In the case of pericardial effusions, the absence or presence of tamponade physiology can dictate the necessity of urgent pericardiocentesis. Our patient developed cardiac tamponade physiology and required pericardiocentesis. In several of the previously reported cases, the pericardial effusion resolved with treatment of hyperthyroidism alone $[2,3,6]$.

Pericardial effusions usually undergo further diagnostic evaluation, as the etiology may be caused by malignancy or tuberculosis. In a United States study, $64 \%$ of pericardial effusions were sanguineous, and malignancy and tuberculosis caused $26 \%$ and $1 \%$, respectively, of the sanguineous pericardial effusions [11]. Seventy percent of malignant pericardial effusions were serosanguineous or sanguineous in an Israeli population [12]. In other studies of sanguineous pericardial effusions, malignancy and tuberculosis accounted for 13$45.6 \%$ and $4-28.6 \%$, respectively, of pericardial effusions [1316]. Our patient appeared to have neither of these etiologies, as culture and cytology of his pericardial fluid did not grow mycobacteria or reveal malignant cells. Furthermore, with treatment for his hyperthyroidism, his pericardial effusion did not recur.

We describe a case of a patient with Graves' disease complicated by sanguineous pericardial effusion, cardiac tamponade, and atrial fibrillation. This condition is rare, with only 10 previously reported cases. Our case is similar to previously reported cases, except that we believe this is the first reported case of sanguineous pericardial effusion involving a heparin infusion. Clinicians should be mindful that although rare, pericardial effusions and cardiac tamponade can be due to hyperthyroidism and that sanguineous pericardial effusion, usually associated with malignancy or tuberculosis, can be due to Graves' disease. Because a pericardial effusion may be sanguineous, the risk of bleeding from the use of anticoagulation for atrial fibrillation should be considered in the setting of hyperthyroidism. The American Heart Association, the American College of Cardiology, and the Heart Rhythm Society recommend administering anticoagulation in the setting of hyperthyroidism based on validated risk factors and risk scoring systems such as $\mathrm{CHA}_{2} \mathrm{DS}_{2}$-VASc (congestive heart failure, hypertension, age $\geq 75$ years [doubled], diabetes mellitus, prior stroke or transient ischemic attack or thromboembolism, vascular disease, age 65 to 74 years, and sex category) $[17,18]$.
Abbreviations
ECG: Electrocardiography
LDH: Lactate dehydrogenase
RBC: Red blood cell
TSH: Thyroid stimulating hormone
T4: Thyroxine
TTE: Transthoracic echocardiography.

\section{Disclosure}

The views expressed in this paper do not communicate an official position of The University of New Mexico.

\section{Competing Interests}

The authors declare that they have no competing interests.

\section{Authors' Contributions}

All authors participated in the drafting, revision, and finalization of the paper and approved the paper for submission and publication.

\section{Acknowledgments}

Cecilia Kieu assisted in the preparation of the figures for this paper.

\section{References}

[1] J. G. Hollowell, N. W. Staehling, W. D. Flanders et al., "Serum $\mathrm{TSH}, \mathrm{T}_{4}$, and thyroid antibodies in the United States population (1988 to 1994): National Health and Nutrition Examination Survey (NHANES III)," Journal of Clinical Endocrinology and Metabolism, vol. 87, no. 2, pp. 489-499, 2002.

[2] N. R. A. Clarke, A. P. Banning, D. J. Gwilt, and A. R. Scott, "Pericardial disease associated with Grave's thyrotoxicosis," Quarterly Journal of Medicine, vol. 95, no. 3, pp. 188-189, 2002.

[3] Y. Khalid, R. Sulaiman, R. Zahir, V. Baskar, and H. N. Buch, "An unusual complication in a patient with Graves' disease," The New Zealand Medical Journal, vol. 124, no. 1341, pp. 69-71, 2011.

[4] P.-Y. Levy, R. Corey, P. Berger et al., "Etiologic diagnosis of 204 pericardial effusions," Medicine, vol. 82, no. 6, pp. 385-391, 2003.

[5] A. Nakata, R. Komiya, Y. Ieki, H. Yoshizawa, S. Hirota, and E. Takazakura, "A patient with Graves' disease accompanied by bloody pericardial effusion," Internal Medicine, vol. 44, no. 10, pp. 1064-1068, 2005.

[6] S. Ovadia, L. Lysyy, and T. Zubkov, "Pericardial effusion as an expression of thyrotoxicosis," Texas Heart Institute Journal, vol. 34, no. 1, pp. 88-90, 2007.

[7] E. Teague, C. J. O’Brien, and N. P. S. Campbell, "Pericardial effusion and tamponade complicating treated graves' thyrotoxocosis," Ulster Medical Journal, vol. 78, no. 1, pp. 56-57, 2009.

[8] M. G. Y. Yu, H. Urbanozo, and M. Fusilero, "Thyrotoxic pericardial effusion complicating graves' disease in pregnancy," Journal of the ASEAN Federation of Endocrine Societies, vol. 30, no. 1, pp. 44-47, 2015.

[9] U. M. Kabadi and S. P. Kumar, "Pericardial effusion in primary hypothyroidism," American Heart Journal, vol. 120, no. 6, part 1, pp. 1393-1395, 1990.

[10] H. H. Parving, J. M. Hansen, S. L. Nielsen, N. Rossing, O. Munck, and N. A. Lassen, "Mechanisms of edema formation in myxedema-increased protein extravasation and relatively slow lymphatic drainage," The New England Journal of Medicine, vol. 301, no. 9, pp. 460-465, 1979.

[11] S. Atar, J. Chiu, J. S. Forrester, and R. J. Siegel, "Bloody pericardial effusion in patients with cardiac tamponade. Is the cause 
cancerous, tuberculous, or latrogenic in the 1990s?" Chest, vol. 116, no. 6, pp. 1564-1569, 1999.

[12] S. Ben-Horin, I. Bank, A. Shinfeld, E. Kachel, V. Guetta, and A. Livneh, "Diagnostic value of the biochemical composition of pericardial effusions in patients undergoing pericardiocentesis," American Journal of Cardiology, vol. 99, no. 9, pp. 1294-1297, 2007.

[13] B. C. Cho, S.-M. Kang, D.-H. Kim et al., "Clinical and echocardiographic characteristics of pericardial effusion in patients who underwent echocardiographically guided pericardiocentesis: Yonsei Cardiovascular Center experience, 1993-2003," Yonsei Medical Journal, vol. 45, no. 3, pp. 462-468, 2004.

[14] C. R. Gibbs, R. D. S. Watson, S. P. Singh, and G. Y. H. Lip, "Management of pericardial effusion by drainage: a survey of 10 years' experience in a city centre general hospital serving a multiracial population," Postgraduate Medical Journal, vol. 76, no. 902, pp. 809-813, 2000.

[15] W. Ma, J. Liu, Y. Zeng et al., "Causes of moderate to large pericardial effusion requiring pericardiocentesis in 140 Han Chinese patients," Herz, vol. 37, no. 2, pp. 183-187, 2012.

[16] J. Sagristà-Sauleda, J. Mercé, G. Permanyer-Miralda, and J. Soler-Soler, "Clinical clues to the causes of large pericardial effusions," American Journal of Medicine, vol. 109, no. 2, pp. 95$101,2000$.

[17] C. T. January, L. S. Wann, J. S. Alpert et al., "American College of Cardiology/American Heart Association Task Force on Practice Guidelines. 2014 AHA/ACC/HRS guideline for the management of patients with atrial fibrillation: a report of the American College of Cardiology/American Heart Association Task Force on Practice Guidelines and the Heart Rhythm Society," Journal of the American College of Cardiology, vol. 64, no. 21, pp. e1-e76, 2014.

[18] G. Y. H. Lip, R. Nieuwlaat, R. Pisters, D. A. Lane, and H. J. G. M. Crijns, "Refining clinical risk stratification for predicting stroke and thromboembolism in atrial fibrillation using a novel risk factor-based approach: The Euro Heart Survey on atrial fibrillation," Chest, vol. 137, no. 2, pp. 263-272, 2010. 


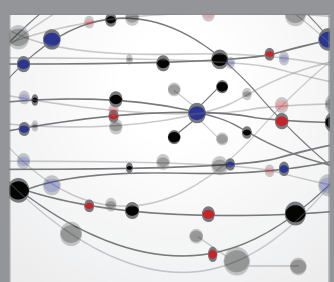

The Scientific World Journal
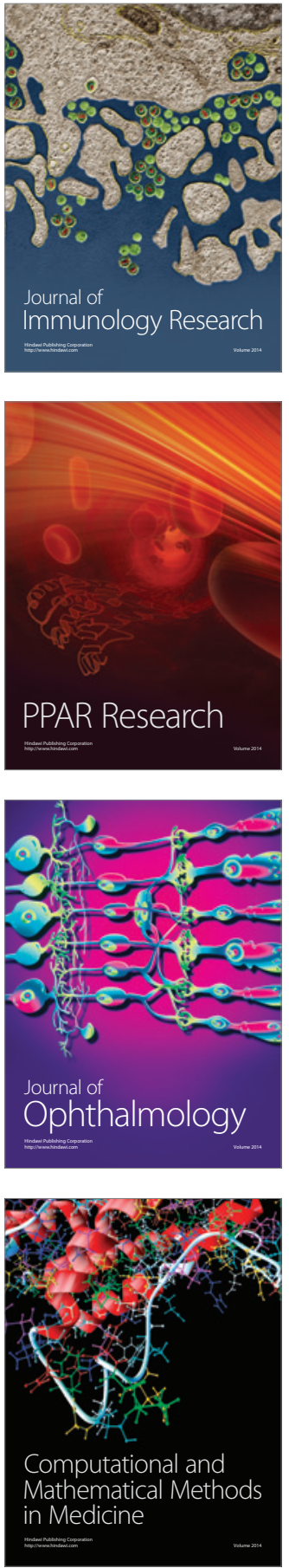

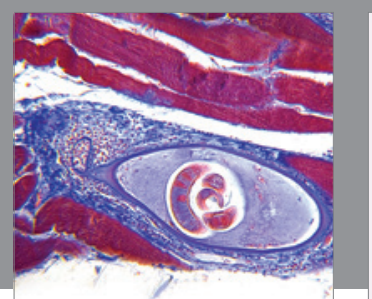

Gastroenterology Research and Practice

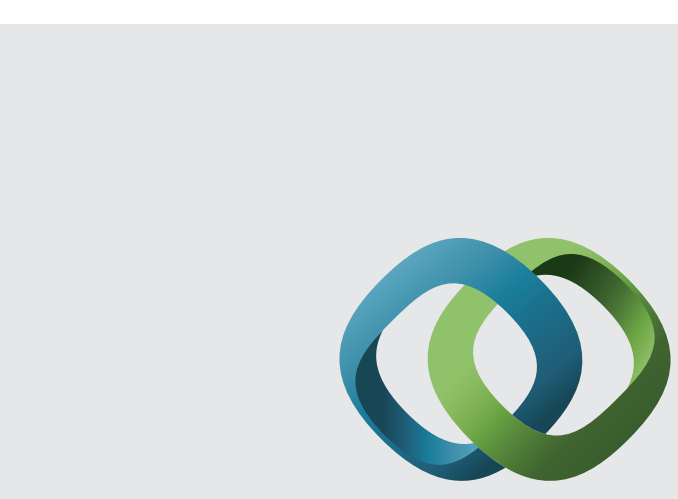

\section{Hindawi}

Submit your manuscripts at

http://www.hindawi.com
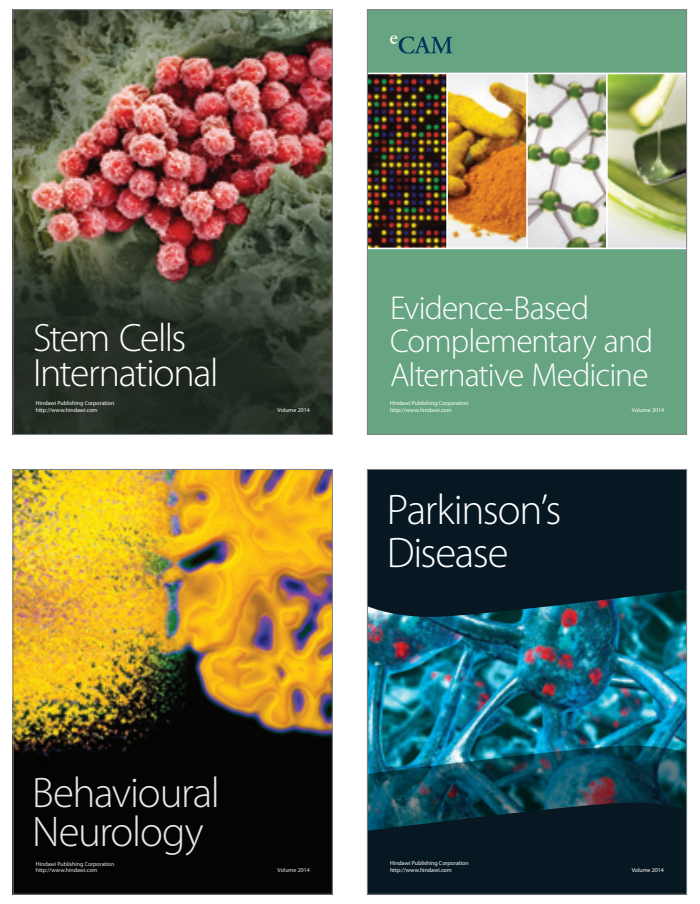
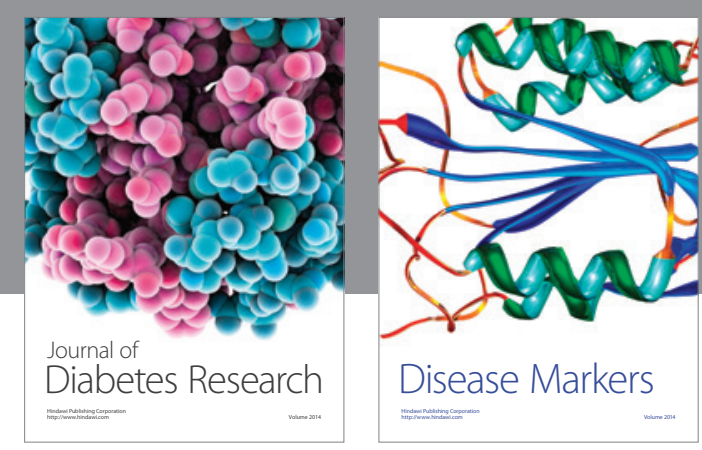

Disease Markers
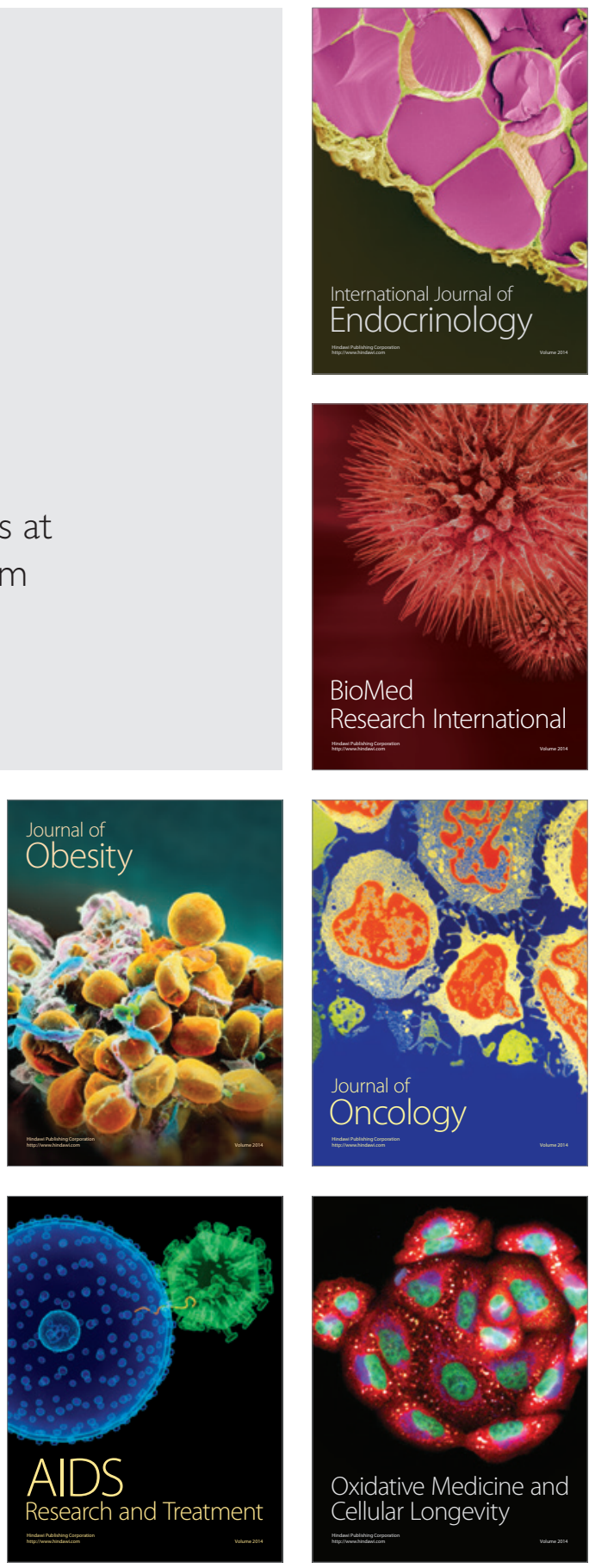